\title{
Hybrid Heat Treatment for Conventionally Treatable Steel Powder Reinforced Age Hardenable Aluminium Alloy Matrix Composites and Mechanical Property Evaluation
}

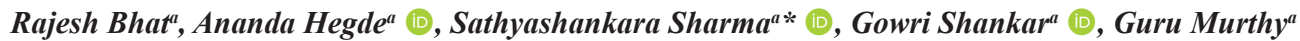 \\ ${ }^{a}$ Manipal Academy of Higher Education, Manipal Institute of Technology, Department of Mechanical \& \\ Manufacturing Engineering, Manipal, 576104, Karnataka, India.
}

Received: August 25, 2021; Revised: November 02, 2021; Accepted: November 29, 2021

\begin{abstract}
The present work is associated with research concentrating on the innovation and use of hybrid heat treatment for eutectoid steel powder ( $0.8 \mathrm{wt} \%)$ reinforced Al-Zn-Mg (Al 7075) alloy composites. Due to high hardness, wear resistance, tensile strength and flexibility modifying heat treatment, heat treatable aluminium metal matrix composites reinforced with heat treatable hard steel particles may be the choice to explore. In this work, an attempt is made to increase hardness and tensile properties to higher levels through hybrid heat treatment, comprising simultaneous treatment to matrix and reinforcement in 3 different routes (Pearlite, Bainite and Martensite) compared to conventional age hardening treatment. SEM images and microhardness distribution witnessed the phase transformation in both matrix and reinforcement. Aging kinetics in conventional age hardening and hybrid treatments is accelerated by the increase in the quantity of reinforcement and increase in aging temperature. The improvement in hardness and tensile strength obtained by conventional age hardening path is further improved by the hybridisation path. Hybridisation route with martensite reinforcement phase shows excellent result in hardness, strength followed by bainite and pearlite path respectively in the decreasing order. In all heat treatment cycles, lower aging temperature marks greater property enhancement compared to higher temperature. Al 7075 with $6 \mathrm{wt} \%$ steel powder reinforced (martensite form) composite showed excellent peak age hardness, tensile strength compared to lesser quantity reinforced composites.
\end{abstract}

Keywords: Aluminium metal matrix composites, microhardness, pearlite, bainite, martensite.

\section{Introduction}

Heat treatment is the controlled heating and cooling process of metal alloys and composites for property modification by precise phase transformation in the solid state with optional change in concentration of alloying elements and shape. Greater improvement in hardness, strength and impact resistance of the material is possible compared to existing methods if the process variables are tailored suitably. There is a provision to alter the heat treatment cycles for the property alterations due to the flexibility available in the process parameters ${ }^{1-4}$. Steels and nonferrous metals are generally subjected to conventional (annealing, normalizing, hardening, tempering etc.) and precipitation supported heat treatments (age hardening and thermomechanical processes). So far in composites (metal matrix composites), only matrix material is subjected to heat treatment and not the reinforcements. Age hardenable alloys like aluminium (2XXX, 7XXX, 6XXX series), Copper (Cu-Be), Titanium (Ti-Al) systems with or without the addition of reinforcement are prone to age hardening with property alterations, like hardness and strength $^{5-12}$.

The age hardenable matrix can be reinforced with heat trea steel powder by stir casting method and matrix definitely subjected to age hardening treatment for property alterations without reinforcement undergoing treatment.

*e-mail: ss.sharma@manipal.edu
At the same time, if reinforcement (steel powder) also undergoes heat treatment simultaneously, the heat treatment has different effect on matrix and reinforcement in a single process may be called as HYBRID heat treatment. In this work, an attempt is made to hybrid heat treatment process by combining age hardening treatment to matrix material and conventional treatments (annealing, normalizing, hardening, austempering etc.) to steel powder at a stretch. Conventional treatments yield different micro constituents (phases) in reinforcement like coarse pearlite, fine pearlite, martensite, bainite etc. with different packing factors of the microconstituents depending on the type of treatments ${ }^{13,14}$. Phase transformation of reinforcement, particle experiences tensile strain within it due to lower packing factor of the transformed microconstituent phases, matrix experiences compressive strain and the resultant is reflected as property alteration, especially, hardness and UTS. At the same time matrix undergoes age hardening treatment and induces tensile strain in the matrix owing to the controlled precipitation of intermetallics (strengthening phases) during heat treatment hybridization. The combined effect of these two processes develops thermal misfit strain between the reinforcement and matrix ${ }^{15}$.

The purpose of the study is to uplift hardness related properties to superior levels through hybrid heat treatment, comprising combined treatment to matrix and reinforcement through novel routes (Pearlite, Bainite and Martensite) 
compared to conventional age hardening treatment. Even though a lot of literature reports are available on non-heat treatable reinforcement reinforced composites, heat treatable reinforcements like, steel powder and alteration in the heat treatment path to have positive impact on both matrix and reinforcement is not yet performed. In this study, such an attempt is made to obtain the composite with superior combination of mechanical properties by newly designed heat treatment procedure. In the area of age hardening composites, this work is the novel approach comprising impact of hybrid heat treatment simultaneously to reinforcement and matrix in the path of property alterations.

\section{Material and Experimental Methods}

\subsection{Matrix and reinforcement materials}

Al 7075 rods are procured from Perfect metal works, Bengaluru in T6 condition. Table 1 shows the composition of matrix material (A1 7075). Steel powder is purchased from Servell Engineers, Mangalore. Table 2 shows the composition of eutectoid steel powder. Table 3 shows properties of Al 7075 alloy and eutectoid steel powder.

\subsection{Preparation of eutectoid steel powder}

Turning operation was performed on eutectoid steel rods to convert into chips using conventional engine lathe. Due to the uninterrupted impact and abrasion by the hardened balls the chips crushes into powders. Measured quantities of eutectoid steel chips were introduced into the mill after preheating to $100^{\circ} \mathrm{C}$. During processing every one-hour interval, these milled particles were removed and were measured for size using different grades of sieves. Process of pulverizing and measuring was repeated till the grain size average of 40-60 micron was achieved.

\subsection{Preparation of composites (Al 7075-eutectoid steel powder MMCs) -Two step stir casting technique}

The methodology adopted in carrying out stir casting for manufacturing of composites is described by the flowchart shown in Figure 1. Composites are fabricated by stir casting technique. Initially, billets of Al 7075 are cut into pieces, placed in a graphite crucible and heated to melt. Melting is continued by increasing the temperature to $750^{\circ} \mathrm{C}$ and soaked isothermally for sufficient time till uniform liquid melt results. The lower melting impurities are removed as slag by introducing small quantity of scum powder. Dry Hexa chloroethane $\left(\mathrm{C}_{2} \mathrm{Cl}_{6}\right), 0.3 \mathrm{wt} \%$ is added to the melt for degasification. Wettability of the reinforcement is enhanced by adding pieces of $\mathrm{Mg}(1 \mathrm{wt} \%)$ to the melt. $\mathrm{Mg}$ also helps

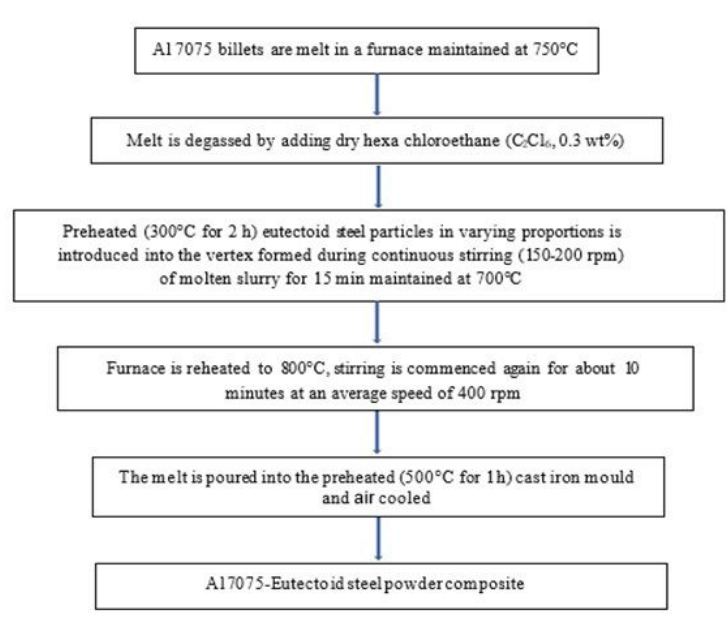

Figure 1. Flow chart of stir casting method used in the study.

Table 1. Composition (wt $\%$ ) of $\mathrm{Al} 7075$ alloy.

\begin{tabular}{ccccccc}
\hline Element & $\mathrm{Cu}$ & $\mathrm{Mn}$ & $\mathrm{Mg}$ & $\mathrm{Zn}$ & $\mathrm{Si}$ & $\mathrm{Al}$ \\
\hline Actual $(\mathrm{wt} \%)$ & 1.35 & 0.08 & 2.21 & 5.67 & 0.4 & Balance \\
\hline Standard (wt $\%)$ & $1.2-1.8$ & $0.1 \mathrm{max}$ & $2-4.5$ & $4-8$ & $0.4 \mathrm{Max}$ & Balance \\
\hline
\end{tabular}

Table 2. Composition (wt \%) of eutectoid steel powder.

\begin{tabular}{ccccccccc}
\hline Elements & $\mathrm{C}$ & $\mathrm{Mg}$ & $\mathrm{Mn}$ & $\mathrm{P}$ & $\mathrm{Cr}$ & $\mathrm{Si}$ & $\mathrm{S}$ & $\mathrm{Fe}$ \\
\hline $\mathrm{wt} \%$ & 0.81 & 0.05 & 0.07 & 0.02 & 0.02 & 0.05 & 0.02 & Balance \\
\hline
\end{tabular}

Table 3. As cast hardness of alloy and composites.

\begin{tabular}{cccccccccc}
\hline Material & & & \multicolumn{1}{c}{ Hardness in Vicker's hardness (HV) scale } & & $\begin{array}{c}\text { Average } \\
\text { hardness } \\
\text { (HV) }\end{array}$ \\
\hline Alloy & 114 & 115 & 113 & 115 & 116 & 116 & 117 & 114 & 115 \\
\hline Composite $2 \%$ & 120 & 122 & 121 & 120 & 122 & 123 & 124 & 120 & 122 \\
\hline Composite $4 \%$ & 126 & 127 & 126 & 128 & 130 & 125 & 126 & 130 & 128 \\
\hline Composite $6 \%$ & 135 & 137 & 134 & 138 & 136 & 134 & 136 & 138 & 136 \\
\hline
\end{tabular}


to strengthen the matrix by the precipitation of secondary intermetallic phases during the controlled heat treatment ${ }^{16}$. The eutectoid steel powder particles are preheated to $300^{\circ} \mathrm{C}$ for $1 \mathrm{~h}$. This process removes all the volatile elements and maintains the powder crispy. The melt is then allowed to cool in air up to $700^{\circ} \mathrm{C}$ to a semi-solid state, further stirred to form the vortex by using a stirrer made of mild steel. During stirring the vortex is formed and eutectoid steel powder in varying proportion $(2,4$ and $6 \mathrm{wt} \%)$ is transferred into the melt in each run. Optimum distribution of the eutectoid steel particles in the alloy can be obtained by maintaining a stirrer in the range of 150-200 rpm for 15 minutes.

The furnace temperature is monitored by a thermocouple. After complete mixing of reinforcement in semisolid state, the furnace is reheated to $750^{\circ} \mathrm{C}$ which is 50 to $60^{\circ} \mathrm{C}$ above the liquidus temperature of Al 7075. Stirring is commenced again for about 10 minutes at an average rpm 400. Composites with uniform reinforcement distribution and minimum casting defects are obtained by employing two stage stir casting technique. Viscosity of the melt in the semisolid state is maintained by adjusting the melt temperature in a semi solid state which in turn reduces the possibility of floating of the eutectoid steel particles. Due to the stirring action the centrifugal force breaks the gas layers around the particle liquid interface, throws the particle which leads to better spread out of molten metal onto the particle surface. Hence dispersivity and wettability are improved. The collective effect of reheating of composite slurry above its liquidus temperature and agitations created during stirring enhances particle distribution by reducing the effect of sedimentation ${ }^{17}$. Finally, the melt is poured into the preheated $(500,1 \mathrm{~h})$ cast iron molds and allowed to cool in air.

The microstructure of all the heat-treated samples are obtained for deciding the quality of castings on particle dispersion in the matrix. The specimens are subjected to micro finishing with conventional polishing and sonication as first step, followed by etching. The samples are polished with various grades of emery papers to mirror like finish without any micro scratches. The polished samples are properly (optimum) etched using Keller's reagent to highlight reinforcement particle and matrix phases. SEM (ModelJEOL JSM 840A) is used to obtain the microstructure of the samples.

Homogeneity in reinforcement dispersion is confirmed by conducting hardness test on five specimens cut from the same casting in the increment of $15 \mathrm{~mm}$ from one end to the other end along the length of casting.

\subsection{Conventional age hardening treatment}

The age hardening treatment is carried out after solution heat treatment (SHT) at $550^{\circ} \mathrm{C}$ for a duration of $2 \mathrm{~h}$ followed by quenching in demineralized water at room temperature. The quenched specimens are artificially aged in the furnace at 100 and $180^{\circ} \mathrm{C}$ for different time intervals and hardness distribution curves are plotted against isothermal aging time. From Al-Zn-Mg ternary phase diagram, as per the ternary eutectic $\mathrm{MgZn}_{2}-(\mathrm{Al})-(\mathrm{Zn})$ phase formation the solutionising temperature is fixed as $550^{\circ} \mathrm{C}$. The harder $\mathrm{MgZn}_{2}$ intermetallic phase found to dissolve completely at $550^{\circ} \mathrm{C}$ during solutionising and sequentially precipitated during aging to strengthen the matrix. It is reported that the samples of Al 7075 and its composites, after solution treatment at $550^{\circ} \mathrm{C}$, exhibits better strength and toughness property combination ${ }^{18}$. The heat treatment cycle used is shown in Figure 2.

\subsection{Hybrid heat treatment}

Step 1 - Conventional heat treatment to ferrous material (steel powder): The composite specimens are heated to 30 $50^{\circ} \mathrm{C}$ above the critical temperature of steel $\left(750^{\circ} \mathrm{C}\right)$ held isothermally for optimum duration of $1 \mathrm{~h}$ and quenched to $550^{\circ} \mathrm{C}$ in a salt bath, held isothermally for $3.5 \mathrm{~h}$ (from Isothermal diagram of eutectoid steel) and air cooled to room temperature ((path 1 in Figure 3)). This is the isothermal annealing treatment for the steel powder to transform as-bought phase into fine pearlite. Fine pearlite is harder and stronger phase compared to as-bought steel phase (coarse pearlite) which strains the matrix material during transformation to alter the properties.

In path 2 (Figure 3 ), the composite specimens are heated to $30-50^{\circ} \mathrm{C}$ above the critical temperature of steel $\left(750^{\circ} \mathrm{C}\right)$ held isothermally for optimum duration of $1 \mathrm{~h}$ and quenched to $350^{\circ} \mathrm{C}$ in a salt bath, held isothermally for $3.5 \mathrm{~h}$ (from

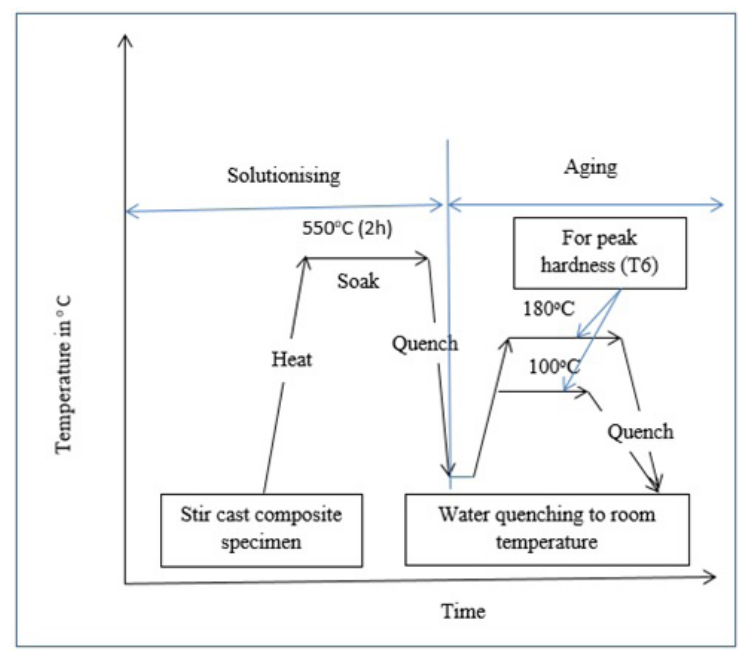

Figure 2. Conventional age hardening treatment.

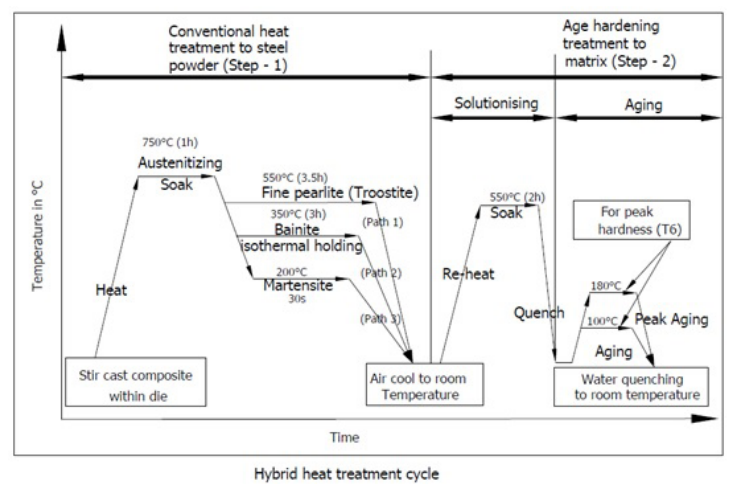

Figure 3. Hybrid heat treatment for the matrix and reinforcement. 
Isothermal diagram of eutectoid steel) and air cooled to room temperature (path 2 in Figure 3). This treatment is conventional austempering for the reinforcement. Now the as-bought steel powder having coarse pearlite structure converts into bainite ${ }^{2}$. This is harder and stronger phase compared to fine pearlite which strains the matrix material to alter the properties. Similarly, another set of specimens are heated to $30-50^{\circ} \mathrm{C}$ above the critical temperature of steel $\left(750^{\circ} \mathrm{C}\right)$ held isothermally for optimum duration of $1 \mathrm{~h}$ and quenched to $200^{\circ} \mathrm{C}$ in a salt bath, held isothermally for $20 \mathrm{~s}$ (from Isothermal diagram of eutectoid steel) and air cooled to room temperature (path 3 ). This treatment is conventional martempering for the reinforcement. Now the as-bought steel powder having coarse pearlite structure converts into martensite ${ }^{2}$. Martensite structure is harder and stronger phase compared to bainite which strains the matrix material better to alter the properties.

Step 2 - Age hardening treatment to matrix material: The partially heat treated (step 1) specimens obtained in the previous step is reheated to $50-100^{\circ} \mathrm{C}$ above the solvus temperature $\left(550^{\circ} \mathrm{C}\right)$ held isothermally for $2 \mathrm{~h}$ to covert twophase matrix material into single phase solid solution and quenched to room temperature to form supersaturated solid solution. On quenching, matrix becomes supersaturated in nature and maintains the crystal structure same as that of high temperature phase without affecting the phases formed (step 1) in reinforcement. The supersaturated matrix phase is reheated below the solvus temperature of the matrix for peak aging (T6 treatment). At the peak aging condition, the entire specimen is quenched to room temperature to arrest the further transformation (over aging). During the step 2, the new phases (pearlite) formed for reinforcement (steel powder) in the step 1 , is not affected while heating below critical temperature i.e., eutectoid temperature $\left(724^{\circ} \mathrm{C}\right)$.

\subsection{Mechanical characterisation}

\subsubsection{Hardness test}

Hardness of the test specimen was deterined using the Micro Vicker's hardness tester. The bar stocks of $10 \mathrm{~mm}$ diameter are cut in to $20 \mathrm{~mm}$ length using power blade hacksaw. The facing operation is carried out on CNC turning centre. Hardness test is carried out using Micro Vicker's hardness testing machine (Akash industries model, A1-Twin) for the test specimens as per ASTM E18-02.

\subsubsection{Tensile test of Al 7075 alloy and composites}

Tensile tests are carried out by following the specifications given in ASTM-E8M standard. Circular cross section specimen with $6 \mathrm{~mm}$ diameter and $24 \mathrm{~mm}$ gauge length is prepared. Tensile test is carried out on Electronic Tensometer. Diameter of specimen is measured using Vernier caliper and cross sectional area is calculated. The load cell value is kept to $20.5 \mathrm{kN}$ and test mode is selected as break. The cross head speed is kept constant at $10 \mathrm{~mm} / \mathrm{min}$, with length increment value of $0.01 \mathrm{~mm}$. The as cast and peak aged specimens are fixed firmly in gripper and load is applied.

\section{Results and Discussions}

\subsection{Microstructure analysis}

The mechanical properties of the composite rely heavily on the degree of dispersion of reinforcement particles. Particulate composites with uniform reinforcement without any agglomeration or sedimentation can be obtained by maintaining suitable rpm of the stirrer and appropriate temperature of the melt. Homogeneity in particle distribution can be studied by microstructural examination using SEM. Figure 4 represents

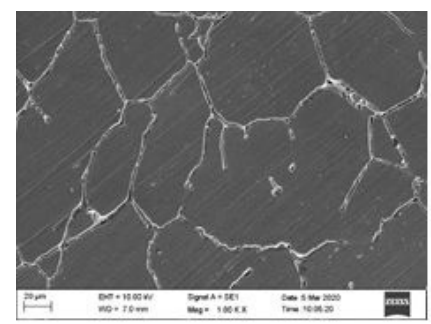

a

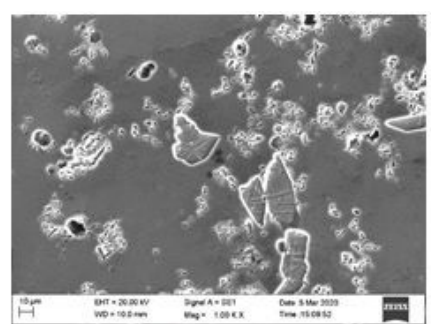

b

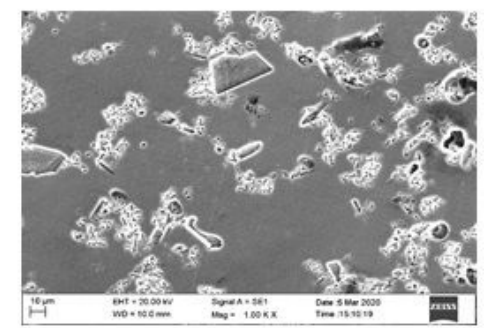

C

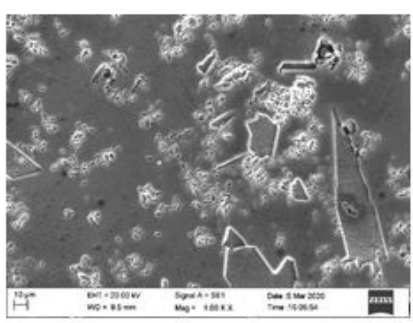

d

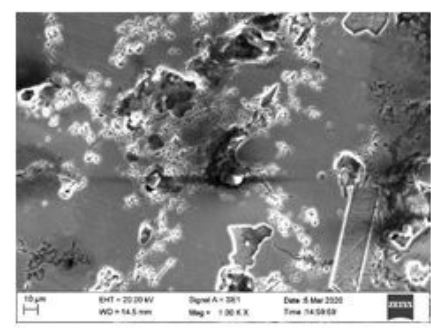

e

Figure 4. Microstructure of as cast Al 7075 alloy and composites in etched condition a) $2 \mathrm{wt} \%$ steel powder b) 4 wt $\%$ steel powder c) 6 wt $\%$ steel powder d) $8 \mathrm{wt} \%$ steel powder e) $8 \mathrm{wt} \%$ steel powder. 
the microstructure of as cast Al 7075 and 2, 4, 6 and $8 \mathrm{wt} \%$ eutectoid steel powder composites in polished and etched condition. The microstructure fairly displays the uniformity of particle distribution in the matrix. Figures 4 shows that dispersion is fairly uniform. Also, porosity or blow holes or clogging of reinforcements or clustering of reinforcements are not observed. Figure $4 \mathrm{e}$ shows agglomeration of steel powder in the SEM image for the composite casting obtained with $8 \mathrm{wt} \%$ steel powder reinforcement. Hence, the reinforcement quantity is restricted to $6 \mathrm{wt} \%$.

\subsection{As cast hardness}

In the as cast condition, the average hardness of the alloy and composites is shown in Table 3 . Table 4 shows the average hardness of the solutionised alloy and composites as measured in Vicker's hardness scale. The average of 8 hardness values is considered for the analysis of experimental result. Hardness distribution in the composite is in the range of $\pm 2 \mathrm{VHN}$ and these castings are accepted as sound casting ${ }^{1}$. The reduction in the hardness values of the solutionised specimen is due to the dissolution of solute rich intermetallics.

Tables 3 and 4, it is seen that increase in quantity of steel powder in the composite increases hardness.

\subsection{Age hardening}

\subsubsection{Aging curves for conventional aging}

The age hardening process is carried out at two different aging temperatures $\left(100\right.$ and $\left.180^{\circ} \mathrm{C}\right)$, the hardness obtained at the different time intervals during aging is noted and graph is plotted as shown in Figures 5a-e. From these graphs it is clear that there are peak values of hardness for both the temperatures for alloy and its respective composites and the hardness value at $100^{\circ} \mathrm{C}$ is higher than the hardness at higher temperature $\left(180^{\circ} \mathrm{C}\right)$. The time taken to reach the peak hardness is more at $100^{\circ} \mathrm{C}$ when compared with $180^{\circ} \mathrm{C}$, because aging kinetics is slow and there is formation of more amount of secondary precipitates at peak aging

Table 4. Solutionised hardness of alloy and composites.

\begin{tabular}{|c|c|c|c|c|c|c|c|c|c|}
\hline \multirow{2}{*}{$\begin{array}{c}\text { Material } \\
\text { Alloy }\end{array}$} & \multicolumn{8}{|c|}{ Hardness in Vicker's hardness (HV) scale } & \multirow{2}{*}{$\begin{array}{c}\begin{array}{c}\text { Average } \\
\text { hardness } \\
(\mathrm{HV})\end{array} \\
106\end{array}$} \\
\hline & 106 & 108 & 104 & 107 & 106 & 105 & 107 & 105 & \\
\hline Composite $2 \%$ & 109 & 110 & 108 & 107 & 108 & 110 & 106 & 108 & 108 \\
\hline Composite $4 \%$ & 109 & 113 & 110 & 113 & 111 & 111 & 109 & 112 & 111 \\
\hline Composite $6 \%$ & 114 & 113 & 117 & 116 & 115 & 113 & 117 & 115 & 115 \\
\hline
\end{tabular}

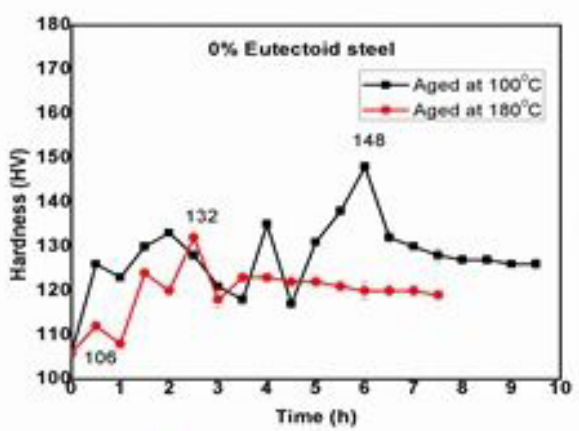

a

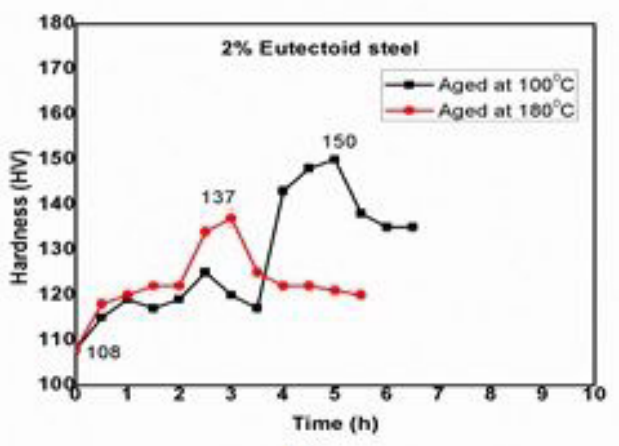

b

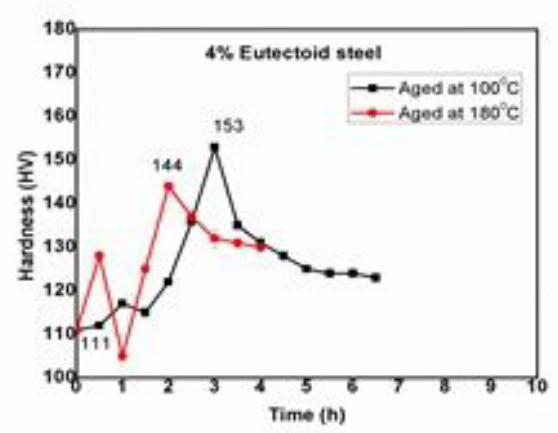

C

Figure 5. Hardness distribution during conventional aging (a) $0 \mathrm{wt} \%$ steel powder (b) $2 \mathrm{wt} \%$ steel powder (c) 4 wt $\%$ steel powder (d) 6 $\mathrm{wt} \%$ steel powder composites. 
condition. The maximum value of hardness $(158 \mathrm{VHN})$ is obtained for $6 \mathrm{wt} \%$ eutectoid steel reinforced composites while aging temperature of $100^{\circ} \mathrm{C}$, aging duration $2.5 \mathrm{~h}$ with $5 \%$ increase in hardness value as compared to that of $2 \mathrm{wt} \%$ reinforcement. As the aging temperature increases, the peak hardness decreases, may be attributed by the reduction in the number of metastable solute rich phases forming during the precipitation of stable intermetallic phase ${ }^{2}$. This statement is well supported by the result obtained.

\subsubsection{Peak hardness in conventional aging}

The effect of the reinforcement on the peak hardness can be clearly seen by the hardness test plot (Figure 6). Hardness of composite increases with the increase in percentage of reinforcement, so it is clear that reinforcement makes the material hard. This is due to the presence of harder eutectoid steel powder. In case of peak aged conditions hardness is maximum during aging at that temperature and time. Excellent peak hardness is obtained at $100^{\circ} \mathrm{C}$ peak aged samples.

\subsection{Hybrid heat treatment}

\subsubsection{Hybrid heat treatment with pearlite as reinforcement}

Hybrid heat treatment consists of conventional treatment to steel powder to alter its phase followed by age hardening treatment. In the first step of conventional treatment the reinforcement steel powder (coarse pearlite) transforms in to fine pearlite. The coarse pearlite present in as cast condition of composite generally has hardness Rc 10-20. When it transforms into fine pearlite by the isothermal holding at $500^{\circ} \mathrm{C}$, enhances its hardness to Rc $40^{19}$. Hence, the casting hardness improves at the end of first step of treatment due to the controlled treatment of the composite, compared to as cast condition. Accordingly, the strain in the lattice changes at the end of first step. During the second step, further controlled treatment of the composite strains the matrix with tensile/ compressive strains as per the packing factor of matrix and reinforcement phases ${ }^{2}$. The complex effect of cooling rate, processing cycle and difference in misfit strain between the materials (aluminium alloy and steel phase) develops enhanced hardness in the composite. There is no much change in the peak aging time in respective categories compared to that of conventional aging (direct) treatment.

The effect of the reinforcement in the pearlitic form on the peak hardness can be clearly seen by the hardness test plot (Figure 7). Up to 5\% increase in the peak hardness values is observed in hybrid treatment with fine pearlitic phase in steel powder as compared to conventional age hardening treatment. The increasing trend of hardness with respect to alteration in aging temperature and reinforcement quantity remains same as that of direct aging treatment (Figure 6).

\subsubsection{Hybrid heat treatment with bainite as reinforcement}

In bainitic phase formation hybrid treatment, the increasing trend of hardness is same as that of the pearlitic trend with increased peak hardness value. By nature, bainite is distorted phase in steel due to its transformation mechanism (both diffusion controlled and shear), witness higher hardness (Rc
40-50). Hence, this transformation induces more strain on the lattice as compared to pearlitic transformation. Since bainite is still fine compared to fine pearlite, the nucleation sites (dislocations) obtained for phase transformation (supersaturated to saturated phases) during the second step of treatment is more, accordingly transformation time for peak aging decreases compared to pearlitic transformation. Also, more number of fine precipitates (solute rich) form in the lattice, which increases the peak hardness value ${ }^{2}$.

The effect of reinforcement in the form of bainite on the hybrid heat treatment behaviour (hardness variation) is shown in Figure 8. Up to $11 \%$ increase in peak hardness is obtained due to the formation of bainite in steel powder as compared to conventional age hardening treatment. Hardness increasing trend in this hybrid treatment also remains same as that of conventional or hybrid treatments (fine pearlite).

\subsubsection{Hybrid heat treatment with martensite as reinforcement}

In martensitic phase transformation hybrid treatment, the increasing trend in hardness remains same as that of fine pearlite and bainite formation hybrid treatments. Martensite is highly distorted phase, exists as supersaturated form in room temperature condition. It is single phase as compared to pearlite and bainite. Pearlite and bainite are

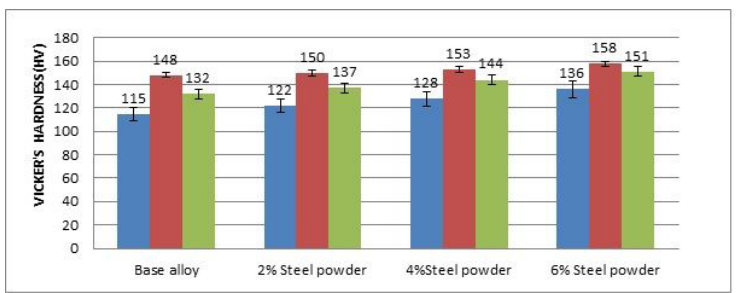

Figure 6. Peak hardness of conventional aged A17075 eutectoid steel composite.

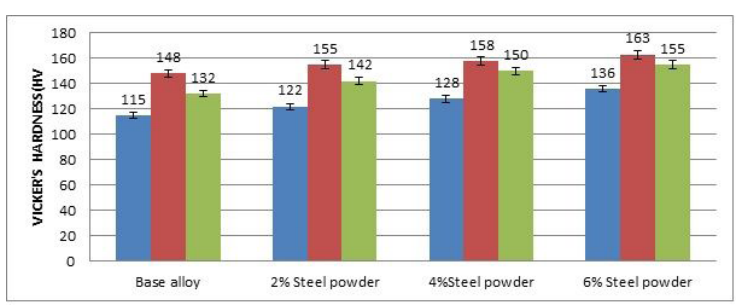

Figure 7. Peak hardness of Al 7075 eutectoid steel composite in hybrid (pearlite) path.

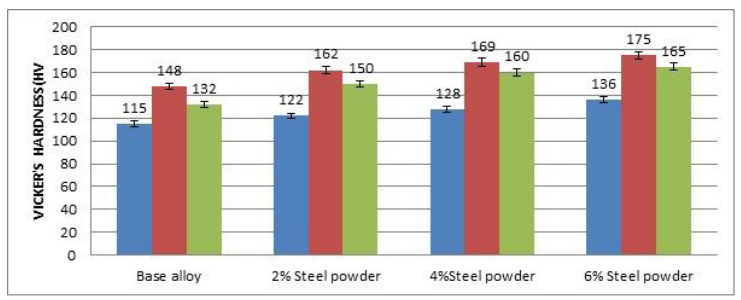

Figure 8. Peak hardness of Al 7075 eutectoid steel composite in hybrid (bainite) path. 
the two-phase mixtures containing ferrite and cementite as micro constituents. Martensite phase in steel forms by shear mechanism without any incubation period and transforms from high temperature austenite phase instantaneously. It is highly expanded phase, occurs with a lot of lattice distortion. As a result of this, hardness of martensite phase increases to Rc 64 in eutectoid steel ${ }^{20}$. Severe distortion of lattice increases the combined hardness of the composite with the increased aging kinetics. The martensite formation is coupled with heavy dislocation density which shoots up the nucleation sites for phase transformation in the second step of hybrid treatment. The greater number of nucleation sites enhances the aging kinetics to reduce the peak aging time.

The effect of the reinforcement in martensite form during hybrid treatment on the peak hardness behaviour can be clearly seen by hardness test plots (Figure 9). As compared to conventional aging, up to $16 \%$ increase in peak hardness is obtained due to the use of hybrid treatment following martensitic path. The Figure 9 clearly shows that increase trend in hardness remains same in conventional age hardening and all hybrid treatments.

\subsubsection{Tensile test results in peak aged condition (Conventional aging)}

From the plot (Figure 10) it is clear that tensile strength of the as cast material increases with the incorporation of the reinforcement (eutectoid steel). In age hardened samples also similar behaviour is observed with increased UTS as compared to as cast composite. The UTS is maximum in case of $100^{\circ} \mathrm{C}$ peak aged samples, in particular, $6 \mathrm{wt} \%$ eutectoid steel reinforced composite material shows highest UTS (402 MPa).

This increase in UTS is due to the presence of eutectoid steel particles acting as barriers to the dislocation movement.

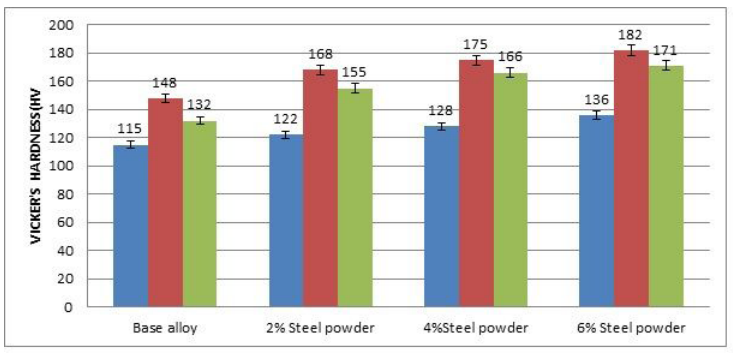

Figure 9. Peak hardness of Al 7075 eutectoid steel composite in hybrid (martensite) path.

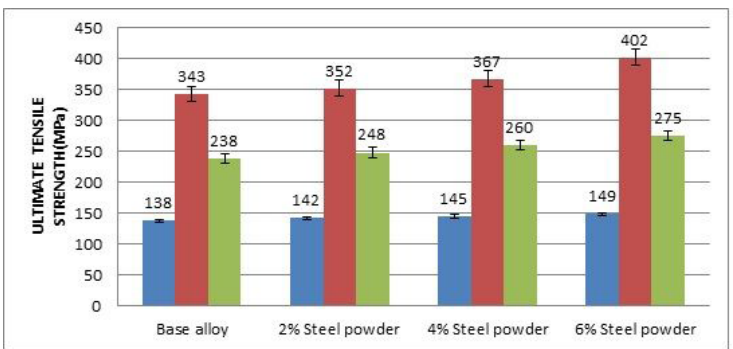

Figure 10. UTS of as cast and peak aged Al 7075 eutectoid steel reinforced composite- Conventional aging.
The increase in strength is due to the combined effect of difference in coefficient of thermal expansion between matrix and eutectoid steel particulates and precipitation behaviour of solute rich secondary phases ${ }^{2}$.

Formation of intermetallic precipitates and presence of steel powder particles in matrix act as the points of obstacles to dislocation movement and hence lead to increase in dislocation density. This increased dislocations at the primary obstacles like, strained reinforcements and intermetallics curtails the physical movement of dislocations under applied load, thereby reducing the extent of plastic flow. This leads to significant improvement in UTS. Lower the aging temperature, more is the number of such primary obstacles for dislocation movement, higher is the strength and hardness ${ }^{21}$ accordingly, UTS increases to high value (402 MPa). The increasing trend in hardness and strength remains same.

\subsubsection{Tensile test results in peak aged condition (Pearlite hybrid treatment)}

From the plot (Figure 11) it is clear that tensile strength of the material increases with the increase in reinforcement (eutectoid steel) in the matrix in as cast and peak aged conditions similar to conventional age hardening treatment. The UTS is maximum (424 MPa) in case of $100^{\circ} \mathrm{C}$ peak aged samples, in particular $6 \mathrm{wt} \%$ eutectoid steel reinforced composite material. The Figure 11 also shows that up to $6 \%$ increase in UTS is obtained with hybrid pearlitic path as compared to conventional age hardening treatment.

This increase in UTS is due to the presence of the coordinated effect of eutectoid steel particles undergoing phase transformation into fine pearlite and intermetallics acting as barriers to the dislocation movement. The increase in strength is due to the combined effect of difference in coefficient of thermal expansion between matrix and eutectoid steel particulates, increase in dislocation density during phase transformation in first step of treatment and precipitation behaviour of solute rich intermetallics ${ }^{8}$.

Formation of intermetallic precipitates with the increased fineness of pearlitic phase act as barrier for dislocation glide and hence lead to strain harden the matrix ${ }^{2}$.

Accordingly, $6 \mathrm{wt} \%$ steel powder reinforced lower temperature peak aged specimen shows higher UTS (424 MPa) compared to the same observed in conventional age hardening treatment.

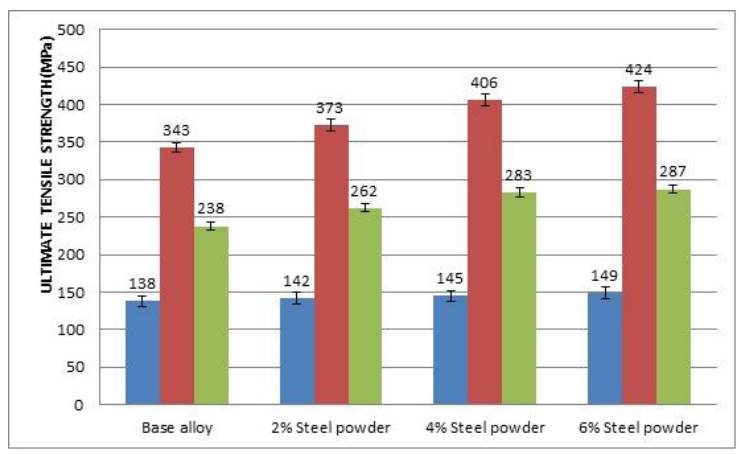

Figure 11. UTS of as cast and peak aged Al 7075 eutectoid steel reinforced composite - Pearlite hybrid treatment. 


\subsubsection{Tensile test results in peak aged condition (Bainite hybrid treatment)}

Bainite condition hybrid treatment shows better UTS compared to that of pearlite condition with a maximum UTS increment of $15 \%$ as compared to conventional age hardening treatment. UTS increasing trend with respect to aging temperature and $w \mathrm{t} \%$ of reinforcement present in the composite remains same as that of conventional age hardening and hybrid treatment pearlite phase. Accordingly, bainitic

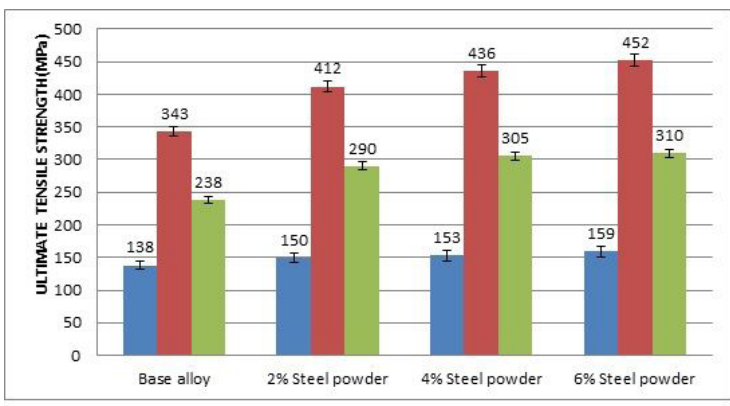

Figure 12. UTS of as cast and peak aged Al 7075 eutectoid steel reinforced composite- Bainite hybrid treatment.

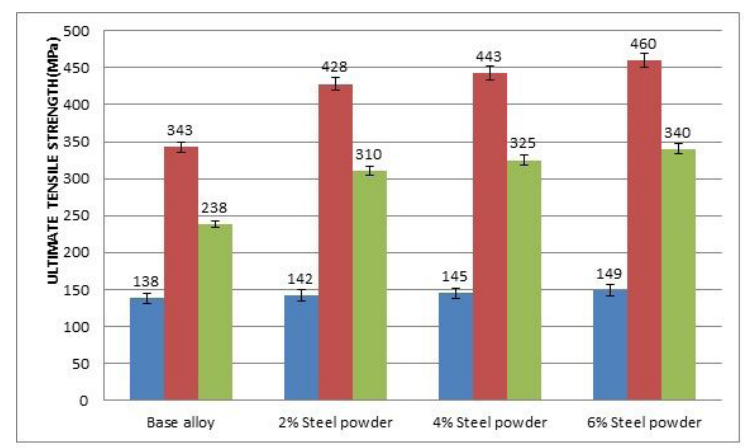

Figure 13. UTS of as cast and peak aged Al 7075 eutectoid steel reinforced composite- Martensite hybrid treatment.
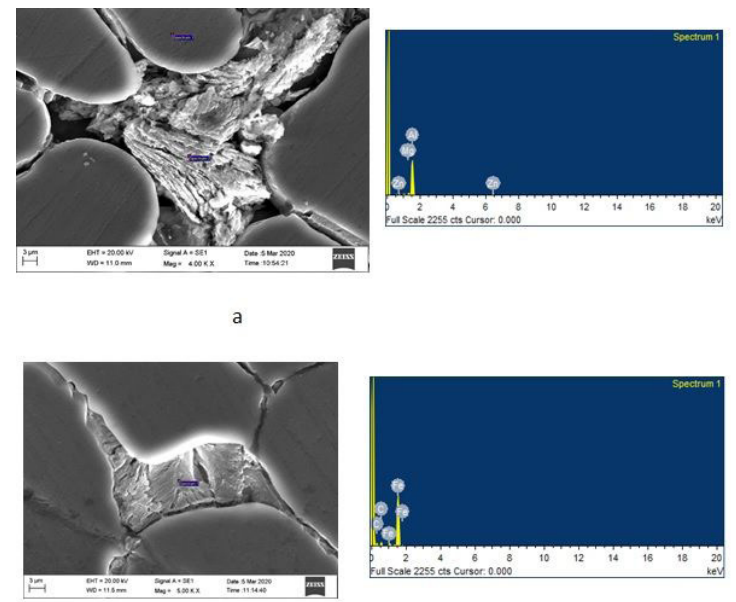

b phase steel reinforcement ( $6 \mathrm{wt} \%)$ with aging temperature $100^{\circ} \mathrm{C}$ shows a maximum UTS $452 \mathrm{MPa}$ (Figure 12). This improvement in the UTS is due to the increase in the hardness of the bainitic phase. As hardness increases tensile property also increases for the $\mathrm{MMC}^{15}$.

\subsubsection{Tensile test results in peak aged condition (Martensite hybrid treatment)}

Hybrid treatment with martensite phase shows similar trend like pearlite or bainite with excellent UTS recording a maximum $460 \mathrm{MPa}$. Since martensite is the hardest non equilibrium phase possible by controlled heat treatment, with low packing density (less than that of BCC Fe, 0.68), expansion of the lattice takes place to strain the lattice which will be reflected in the form of hardness and tensile strength improvement. Accordingly, martensite phase steel reinforcement $(6 \mathrm{wt} \%)$ composite with aging temperature of $100^{\circ} \mathrm{C}$ shows maximum UTS among the group of composites, recording $460 \mathrm{MPa}$, which is $33 \%$ improvement compared to that of conventional age hardening treatment as shown in Figure 13.

\subsection{Confirmation test for hybrid treatment}

For the confirmation of hybrid treatment, the evidence of microstructure in conventional and three different hybrid treatment paths as well as corresponding microhardness in the specimen are analyzed.

\subsection{Microstructural study of peak aged composites}

The SEM image of polished and etched samples of peak aged conventionally treated and hybrid method heat treated Al 7075-6 wt \% steel powder composite specimens are shown in Figure 14. Figure 14a shows conventionally peak aged specimen with EDAX at regions 1 and 2, where region 1 represents base alloy location, showing existence of $\mathrm{Al}, \mathrm{Zn}$ and $\mathrm{Mg}$ elements. Figure 14a, region 2 shows the steel reinforcement (representing $\mathrm{Fe}$ and $\mathrm{C}$ elements) with well-defined lamellar structure (coarse), known as
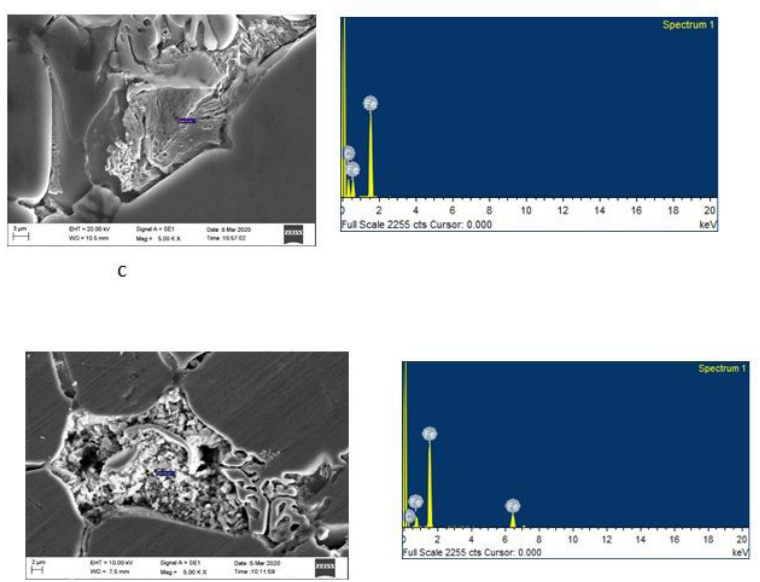

d

Figure 14. SEM micrographs with EDAX of a) conventionally b) hybrid pearlite formed c) hybrid bainite formed d) hybrid martensite formed peak aged $\mathrm{Al} 7075-6 \mathrm{wt} \%$ steel powder composite. 
coarse pearlite. Figure 14b shows reinforcement as lamellar structure, known as fine pearlite, where lamellae gap is very less ${ }^{2}$. This phase is harder than the as cast reinforcement phase (coarse pearlite) shown in Figure 1a. EDAX of region marked in the microstructure shows that reinforcement is steel (representing Fe and C elements).

Figure 14c shows reinforcement in feathery form, known as bainite ${ }^{22}$. This phase is harder than pearlite and marginally softer than martensite. EDAX of region marked in the microstructure also shows that reinforcement is steel (representing Fe and C elements). Figure 14d shows reinforcement structure as randomly oriented laths. This is a typical microstructure of martensite. This phase is harder than bainite ${ }^{2}$. EDAX of region marked in the microstructure also shows that reinforcement is steel (representing Fe and C elements).

\section{Conclusions}

Al 7075 steel powder reinforced composites are stir cast and subjected to conventional and hybrid treatments with improvement in hardness related properties. Microstructure result of the cast composite restricted the steel powder addition to $6 \mathrm{wt} \%$. The hybrid heat treatments comprising simultaneous treatment to matrix and reinforcement in pearlite, bainite and martensite paths show considerable enhancement in hardness and tensile properties compared to conventional age hardening. SEM images confirmed the phase transformation in steel powder to support the mechanical test out comes. In composites, as the $\mathrm{wt} \%$ of steel powder increases peak hardness values of composites in respective heat treatment condition increases. Hybridisation route with martensite path shows excellent result in hardness and strength followed by bainite and pearlite path respectively in the decreasing order. Al 7075 with $6 \mathrm{wt} \%$ steel powder (martensite form) composite showed superior peak age hardness and tensile strength values among the group.

\section{References}

1. Bayazida S M, Farhangi H, Asgharzadeh H, Radan L, Ghahramani A, Mirhaji A. Effect of cyclic solution treatment on microstructure and mechanical properties of friction stir welded 7075 Al alloy. Mater Sci Eng. 2014;649:293-300.

2. Rajan T V, Sharma C P, Sharma A. Heat treatment: principles and techniques. New Delhi: PHI Learning; 2012.

3. Li JF, Peng ZW, Li CX, Jia ZQ, Chen WJ, Zheng ZQ. Mechanical properties, corrosion behavior and microstructures of 7075 aluminium alloy with various aging treatment. Trans Nonferrous Met Soc China. 2008;18:755-62.

4. Qamar SZ. Effect of heat treatment on mechanical properties of H11 tool steel. J Achiev Mater Manuf Eng. 2009;35(2):115-120.
5. Rebba B, Ramanaiah N. Evaluation of mechanical properties of aluminium alloy (Al-7075) reinforced with molybdenum disulphide $\left(\mathrm{MoSi}_{2}\right)$ metal matrix composites. Procedia Materials Science. 2014;6:1161-9.

6. Surappa MK. Aluminium matrix composites: challenges and opportunities. Sadhana. 2003;28(1-2):319-34.

7. Sharma P, Chauhan G, Sharma N. Production of AMC by stir casting-an overview. J Contemp. 2011;2(1):23-46.

8. Clark R, Coughran B, Traina I, Hernandez A, Scheck T, Etuk C. On the correlation of mechanical and physical properties of 7075-T6 Al alloy. Eng Fail Anal. 2005;12:520-6.

9. Mathur S, Barnawal A. Effect of process parameter of stir casting on metal matrix composites. Int J Sci Res. 2013;2(12):395-8.

10. Das S, Das S, Das K. Ageing behavior of Al- $4.5 \mathrm{wt} \% \mathrm{Cu}$ matrix alloy reinforced with $\mathrm{Al}_{2} \mathrm{O}_{3}$ and $\mathrm{ZrSiO}_{4}$ particulate varying particle size. J Mater Sci. 2006;41(16):5402-6.

11. Kerti I, Toptan F. Microstructural variations in cast $\mathrm{B}_{4} \mathrm{C}$ -reinforced aluminium matrix composites (AMCs). Mater Lett. 2008;62(8):1215-8.

12. Toptan F, Kilicarslan A, Karaaslan A, Cigdem M, Kerti I. Processing and microstructural characterisation of AA 1070 and AA 6063 matrix $\mathrm{B}_{4} \mathrm{C}_{\mathrm{p}}$ reinforced composites. Mater Des. 2010;32:S87-91.

13. Mahathaninwonga N T, Plookphola J, Wannasin J, Wisutmethangoon S. T6 heat treatment of rheocasting $7075 \mathrm{Al}$ alloy. Mater Sci Eng. 2012;532:91-9.

14. Mandal D, Ghosh M, Pal J, De PK, Chowdhury SG, Das SK, et al. Effect of austempering treatment on microstructure and mechanical properties of high-Si steel. J Mater Sci. 2009;44(4):1069-75.

15. Uvaraja VC, Natarajan N, Sivakumar K, Jegadheeshwaran S, Sudhakar S. Tribological behavior of heat-treated Al 7075 metal matrix composites. Indian J Eng Mater Sci. 2015;22(1):51-61.

16. Reda Y, Abdel-Karim R, Elmahallawi I. Improvements in mechanical and stress corrosion cracking properties in Al 7075 via retrogression and reaging. Mater Sci Eng. 2008;485(12):468-75.

17. Kanayo K, Kazeem A, Sanusi O. Microstructural characteristics, mechanical and wear behaviour of aluminium matrix hybrid composites reinforced with alumina, rice husk ash and graphite. Engineering Science and Technology. 2015;18:416-22.

18. Zou X, Yan H, Chen X. Evolution of second phases and mechanical properties of $7075 \mathrm{Al}$ alloy processed by solution heat treatment. Trans Nonferrous Met Soc China. 2017;27:2146-55.

19. Herian J, Aniołek K, Cieśla M, Skotnicki G. Shaping the structure during rolling and isothermal annealing, and its influence on the mechanical characteristics of high-carbon steel. Mater Sci Eng A. 2014;608:149-54.

20. Bhagyalaxmi SS, Kini V. Effect of heat treatment and mechanical characterization of AISI 4140 steel. Int J Mech Prod. 2018;8(9):603-10.

21. Rebba B, Ramanaiah N. Evaluation of mechanical properties of aluminium alloy (Al-7075) reinforced with molybdenum disulphide $\left(\mathrm{MoSi}_{2}\right)$ metal matrix composites. Procedia. 2014;6:1161-9.

22. Avner SH. Introduction to physical metallurgy. New York: McGraw-Hill; 2012. 